\title{
Dimensional synthesis of a spherical parallel manipulator based on the evaluation of global performance indexes
}

\author{
Lisandro J. Puglisi , Roque J. Saltaren, Hector A. Moreno, Pedro F. Cárdenas, Cecilia Garcia, Rafael Aracil
}

\begin{abstract}
A B S T R A C T
In this work, the dimensional synthesis of a spherical Parallel Manipulator (PM) with a 3PSU-1S kinematic chain is presented. The goal of the synthesis is to find a set of parameters that defines the PM with the best performance in terms of workspace capabilities, dexterity and isotropy. The PM is parametrized in terms of a reference element, and a non-directed search of these parameters is carried out. First, the inverse kinematics and instantaneous kinematics of the mechanism are presented. The latter is found using the screw theory formulation. An algorithm that explores a bounded set of parameters and determines the corresponding value of global indexes is presented. The concepts of a novel global performance index and a compound index are introduced. Simulation results are shown and discussed. The best PMs found in terms of each performance index evaluated are locally analyzed in terms of its workspace and local dexterity. The relationship between the performance of the PM and its parameters is discussed, and a prototype with the best performance in terms of the compound index is presented and analyzed.
\end{abstract}

\section{Introduction}

The synthesis of a parallel mechanism (PM) can be divided into two main topics, the definition of the mechanical architecture (Structural Synthesis), and the determination of the dimensions of its elements (Dimensional Synthesis).

The first is related to the number, type and relative position of joint axes of the PM, which can be obtained by several systematic approaches [1-3]. Even though the pattern of movement, the actuators and the degrees of freedom of the PM are defined during the structural synthesis, the incorrect dimensioning of its elements will result in a mechanism with poor performance. Therefore, the correct design of a PM must unequivocally determine the best geometry that satisfies the design requirements [4].

For the dimensional synthesis of a PM, the mechanism has to be modeled and the dimensions that are most appropriate for the task according to the criteria adopted for the evaluation of its performance, have to be found.

Some authors base the dimensional synthesis on the maximization of a performance index related to the Jacobian matrix. The
Condition Index and the Global Conditioning Index (GCI) are perhaps the most known and used indexes for the design and evaluation of a mechanism [5-9]. However, it is often used for the definition of a cost function and an optimization problem. In [10], the optimal kinematic design is formulated as a non-linear programming problem of a cost function based on the media and standard deviation of the condition number of the Jacobian matrix.

Since the optimization problem of a cost function is quite complex, genetic algorithms (GA) or other search methods during the synthesis are often used. In [11], the optimization of the parameters of a three-legged 6DoF Haptic device is carried out by the minimization of an objective function, which is defined by the minimum of the global design indexes implementing a GA for a global optimization. GA is also implemented in [12] to determine the optimal configuration of a 3UPU pure translation and a 3UPS pure orientation PM. It also implements Monte Carlo's method to determine the performance indexes. In [13] a controlled random search algorithm is presented in order to solve the constrained non-linear optimization problem that maximizes the effective regular workspace of a Delta and a Stewart-Gough robot.

In this work, the dimensional synthesis for a novel spherical $\mathrm{PM}$ is presented. The PM is parametrized considering one of its elements as reference, which provides a scalable solution for a real application. A non-directed search of these parameters is carried out in order to find the relationship between the parameters that guarantee a good performance in terms of a novel compound global index that integrates information of its workspace capabilities, dexterity and isotropy. The results obtained during the simulations are implemented into a real prototype and its real physical characteristics are presented. It also shows that the design criteria 


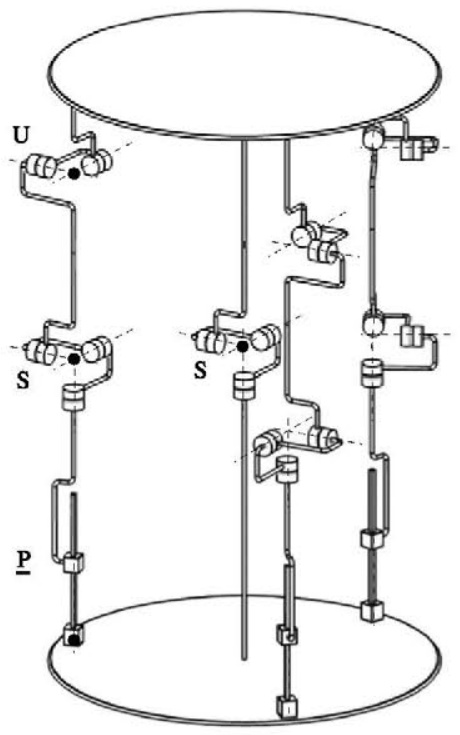

Fig. 1. 3PSU-1S PM.

cannot depend on the evaluation of only one global performance index.

This paper is organized as follows: first, a brief introduction to 3PSU-1S spherical parallel manipulator and its kinematic modeling are presented. Then, the methodology applied is completely detailed and summed up in a descriptive flow chart. It is followed by the results from simulations, which are presented and further discussed. Finally the conclusions are stated.

\section{3PSU-1S spherical parallel manipulator}

The 3PSU-1S is a four-legged parallel mechanism, where the passive leg imposes the spherical movement pattern, controlled by three PSU type legs with the prismatic pair actuated (see Fig. 1).

Since the active legs are placed over the vertices of an equilateral triangle, the geometry of the mechanism can be fully characterized by the length of the links $L_{1}, L_{2}, L_{3}$ and $L_{4}$, and the radius $R_{B}$ and $R_{M}$ of the circumscribed circle of the equilateral triangles of the base and moving platform respectively, see Fig. 2(a).

\subsection{Inverse kinematics}

Let us attach a fixed reference frame $O_{x y z}$ at the center of rotation of the passive leg as presented in the schematic wire diagram of the mechanism in Fig. 2(a).

The link $L_{3}$ of the ith-leg is connected to the moving platform by a universal joint, thus the opposite extreme of this link $\left(B_{i}=\right.$ $\left.\left[B_{i_{x}}, B_{i_{y}}, B_{i_{z}}\right]\right)$ can only move over the surface of the sphere $\zeta_{i}$ with center in $C_{i}=\left[C_{i x}, C_{i y}, C_{i_{z}}\right]$ and radius $L_{3}$ (see Fig. 2(b)), defined according to (1).

$\left(B_{i_{x}}-C_{i_{x}}\right)^{2}+\left(B_{i_{y}}-C_{i_{y}}\right)^{2}+\left(B_{i_{z}}-C_{i_{z}}\right)^{2}=L_{3}^{2}$.

$B_{i}$ also belongs to line $I_{i}$, whose direction vector is associated to the axis of the prismatic actuator, defined by (2),

$l_{i}: B_{i}=A_{i}+\lambda_{i} \hat{\boldsymbol{l}}_{i}$

where $\hat{\boldsymbol{l}}_{i}$ is the unit vector pointing along the direction of line $l_{i}$, and is parallel to the $\boldsymbol{z}$ axis (i.e. $\hat{\boldsymbol{t}}_{i}=[0,0,1]$ ). Expanding (2), it can be demonstrated that $B_{i_{x}}=A_{i_{x}}$ and $B_{i_{y}}=A_{i_{y}}$. Taking this result into (1), $B_{i_{z}}$ can be found according to (3).

$B_{i_{z}}=C_{i_{z}} \pm \sqrt{L_{3}^{2}-\left(A_{i_{x}}-C_{i_{x}}\right)^{2}-\left(A_{i_{y}}-C_{i_{y}}\right)^{2}}$. a
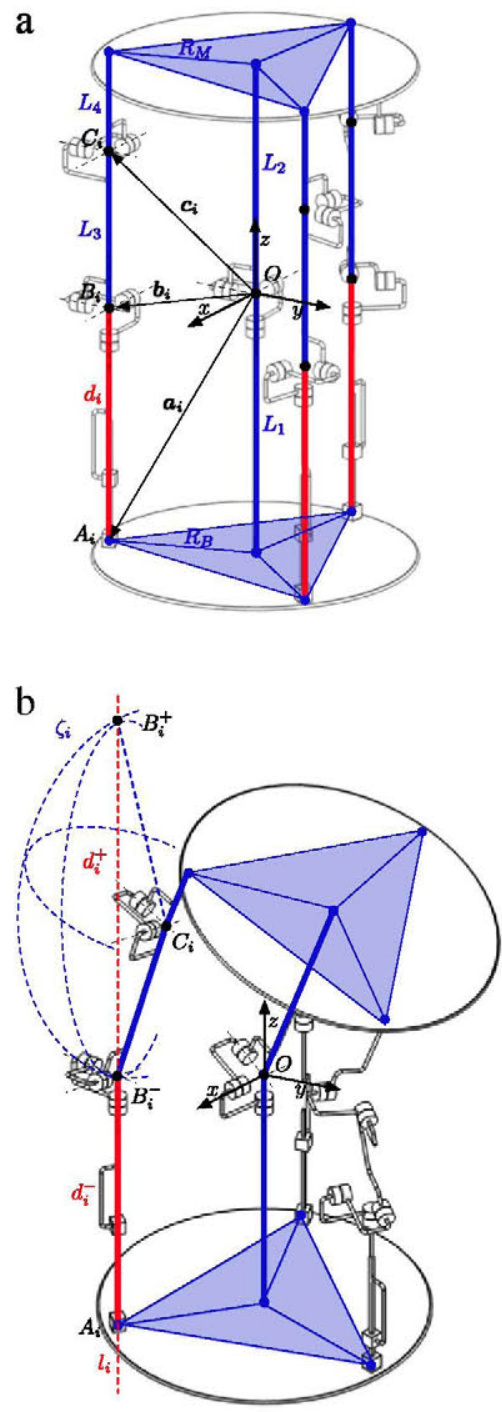

Fig. 2. Schematic wire diagram of the 3PSU-1S.

Given the nature of the square root operator, then two possible solutions can be obtained $B_{i_{z}}^{-}, B_{i_{z}}^{+}$when selecting the - or + sign in (3) respectively. However, the $B_{i z}^{+}$solution has to be discarded, because it leads to collisions between the legs and the moving platform.

Then, the state of the prismatic actuator is given by (4). $\lambda_{i}=B_{i_{z}}-A_{i_{z}}$.

\subsection{Screw based Jacobian matrix}

The traditional process for finding the instantaneous kinematics of a parallel mechanism consists of differentiating the inverse kinematic equation. However, this process is tedious and may lead to possible errors. A much better methodology is the use of screw theory and the concept of reciprocal screws [14]. They provide a better geometrical insight into the problem and allow the precise and complete analysis of singularities of the mechanism $[15,16]$.

Therefore, let us define an instantaneous reference frame $O_{x^{\prime} y^{\prime} z^{\prime}}^{\prime}$ which is instantaneously coincident and parallel to the reference frame $O_{x y z}$, and express all the twists of the mechanism with respect to this instantaneous reference frame. All the twists associated to the joints of the ith-leg of the 3PSU-1S mechanism are shown in Fig. 3, where $\$_{j, i}$ is the jth-twist associated to the 
jth-joint of the ith-leg, and $\boldsymbol{u}_{j, i}$, is the unit vector associated to the corresponding twist. The instantaneous reference frame $O_{x^{\prime} y^{\prime} z^{\prime}}^{\prime}$ is not shown for graph clarity.

According to [17], the resulting twist for the end effector $\left(\$_{e f}\right)$ can be found as the linear combination of the twists associated to each joint of a leg given by (5),

$$
\begin{aligned}
\$_{\text {ef }} & =\left[\begin{array}{c}
\omega_{\text {ef }} \\
\boldsymbol{v}_{O^{\prime}}
\end{array}\right] \\
& =\dot{q}_{1, i} \hat{\$}_{1, i}+\dot{q}_{2, i} \hat{\$}_{2, i}+\dot{q}_{3, i} \hat{\$}_{3, i}+\dot{q}_{4, i} \hat{\$}_{4, i}+\dot{q}_{5, i} \hat{\$}_{5, i}+\dot{q}_{6, i} \hat{\$}_{6, i}
\end{aligned}
$$

where $\omega_{e f}=\left[\begin{array}{lll}\omega_{x}, & \omega_{y}, & \omega_{z}\end{array}\right]^{T}$, is the angular velocity of the end effector, $\boldsymbol{v}_{O^{\prime}}=\left[\begin{array}{lll}v_{O x^{\prime}}, & v_{O y^{\prime}}, & v_{O z^{\prime}}\end{array}\right]^{T}$ is the linear velocity of a point that belongs to the end effector expressed at the origin of the instantaneous reference frame, $\hat{\$}_{j, i}$ is the unitary twist associated to the jth-joint of the ith-leg, and $\dot{q}_{j, i}$ is its corresponding intensity.

It must be noticed in (5) that since the pattern of movement of the mechanism is governed by the spherical joint of the passive leg, no translation for the end effector is allowed, therefore $\boldsymbol{v}_{O^{\prime}}=$ $\left[\begin{array}{lll}0, & 0, & 0\end{array}\right]^{T}$.

Using the reciprocal screw's property, all the twists of the passive joints can be eliminated from (5). In particular, the reciprocal screw to any spherical joint and universal joint, is a $\$_{0}$ screw that passes through the center of the joint. Therefore, the reciprocal screw for each leg is a $\$_{0}$ that passes through the center of the universal joint and the center of the spherical joint, and given by (6),

$\hat{\$}_{r, i}=\left[\begin{array}{c}\boldsymbol{u}_{r, i} \\ \boldsymbol{b}_{i} \times \boldsymbol{u}_{r, i}\end{array}\right]$

where $\boldsymbol{u}_{r, i}=\left(\boldsymbol{c}_{i}-\boldsymbol{b}_{i}\right) /\left\|\boldsymbol{c}_{i}-\boldsymbol{b}_{i}\right\|$. Taking the reciprocal product $\hat{\$}_{r, i} \circ \$_{e f},(5)$ is reduced to (7).

$\hat{\$}_{r, i} \circ \$_{e f}=\dot{q}_{1, i}\left(\hat{\$}_{r, i} \circ \hat{\$}_{1, i}\right)$.

Considering the reciprocal product operator $\Pi=\left[\begin{array}{ll}\varnothing & I \\ I & \varnothing\end{array}\right]$, where $I$ is the $3 \times 3$ identity matrix and $\varnothing$ is the $3 \times 3$ zero matrix, (7) can be expressed as follows [1]:

$\left[\Pi \hat{\$}_{r, i}\right]^{T} \hat{\$}_{e f}=\dot{q}_{1, i}\left(\left[\Pi \hat{\$}_{r, i}\right]^{T} \hat{\$}_{1, i}\right)$,

where $\hat{\$}_{1, i}=\left[\begin{array}{ll}\mathbf{0}, & \boldsymbol{u}_{1, i}\end{array}\right]^{T}$ is the twist $\$_{\infty}$ associated to the prismatic joint.

Replacing $\hat{\$}_{1, i}$ and $\$_{\text {ef }}$ in (8), and expanding for legs $i=1,2,3$, the velocity equation of the mechanism can be found according to (9).

$$
\begin{aligned}
& {\left[\begin{array}{c}
\left(\boldsymbol{b}_{1} \times \boldsymbol{u}_{r, 1}\right)^{T} \\
\left(\boldsymbol{b}_{2} \times \boldsymbol{u}_{r, 2}\right)^{T} \\
\left(\boldsymbol{b}_{3} \times \boldsymbol{u}_{r, 3}\right)^{T}
\end{array}\right] \omega_{e f}} \\
& =\left[\begin{array}{ccc}
\boldsymbol{u}_{r, 1}^{T} \boldsymbol{u}_{1,1} & 0 & 0 \\
0 & \boldsymbol{u}_{r, 2}^{T} \boldsymbol{u}_{1,2} & 0 \\
0 & 0 & \boldsymbol{u}_{r, 3}^{T} \boldsymbol{u}_{1,3}
\end{array}\right]\left[\begin{array}{l}
\dot{q}_{1,1} \\
\dot{q}_{1,2} \\
\dot{q}_{1,3}
\end{array}\right] .
\end{aligned}
$$

Defining $J_{x}$ and $J_{q}$ as follows,

$J_{x}=\left[\begin{array}{c}\left(\boldsymbol{b}_{1} \times \boldsymbol{u}_{r, 1}\right)^{T} \\ \left(\boldsymbol{b}_{2} \times \boldsymbol{u}_{r, 2}\right)^{T} \\ \left(\boldsymbol{b}_{3} \times \boldsymbol{u}_{r, 3}\right)^{T}\end{array}\right]$,

$J_{q}=\left[\begin{array}{ccc}\boldsymbol{u}_{r, 1}^{T} \boldsymbol{u}_{1,1} & 0 & 0 \\ 0 & \boldsymbol{u}_{r, 2}^{T} \boldsymbol{u}_{1,2} & 0 \\ 0 & 0 & \boldsymbol{u}_{r, 3}^{T} \boldsymbol{u}_{1,3}\end{array}\right]$,

the velocity equation of the mechanism can be expressed as:

$J_{x} \omega_{e f}=J_{q} \dot{\boldsymbol{q}}$,

where $\dot{\boldsymbol{q}}=\left[q_{1,1}, \quad q_{1,2}, \quad q_{1,3}\right]^{T}$.

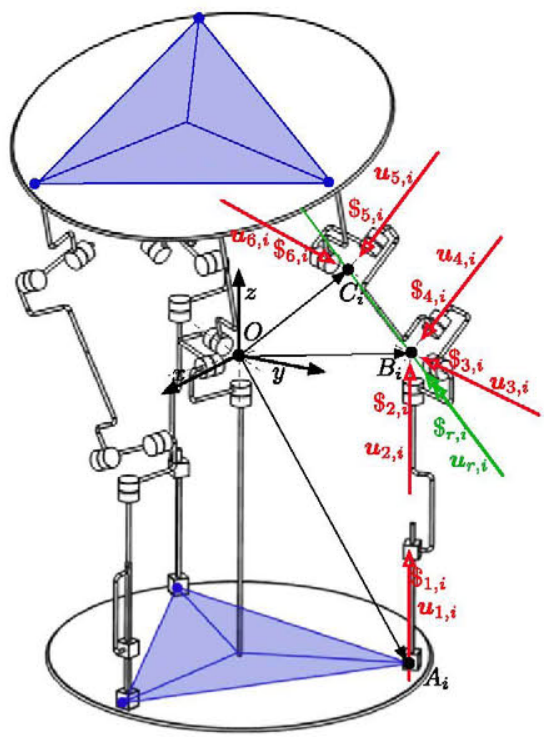

Fig. 3. Screws associated to the 3PSU-1S PM.

If the inverse matrix of $J_{q}$ exists, the velocity equation of the mechanism can be written in terms of the overall Jacobian matrix $J=J_{q}^{-1} J_{x}$, as follows:

$J \omega_{e f}=\dot{\boldsymbol{q}}$

\section{Dimensional synthesis methodology}

The methodology proposed is based on the parametrization of the PM in terms of the length of the elements $L_{1}, L_{2}, L_{3}, L_{4}$, $R_{B}$ and $R_{M}$, and their normalization according to a geometric characteristic. This consideration allows us to have a dimensionless analysis, which permits a scalable solution. A bounded set of these parameters is defined and the algorithm analyzes all the PMs in terms of the global performance indexes presented below. For the evaluation of the global performance indexes (GPI), the workspace of each PM is discretized into nodes. A non-oriented search for the parameters where the PM has the best performance in terms of a compound index is performed.

A schematic flow chart of this method is presented in Fig. 4. As it can be observed, the algorithm can be divided into three main tasks:

1. Parameter generation: it generates the different sets of parameter that describe a unique mechanism.

2. Mechanism evaluation: for every generated set of parameters, the geometric relations of the PM are established and the performance of the PM is evaluated over its discretized workspace. These steps are detailed in the following subsections.

3. Data processing: the algorithm shows the relationships between each GPI and the PM's geometry. It finds the set of parameters that maximizes each of the GPI evaluated. It also evaluates the following compound index:

$C=\beta W S I\left(\sum_{i}^{n} \alpha_{i} G P I_{i}\right)$,

which is intended to integrate into a single index the workspace and dexterity characteristic of the PM. $\beta$ and $\alpha_{i}$ are ponderation factors.

The PMs with maximum performance found are analyzed considering their local dexterity behavior and their workspace capabilities. 


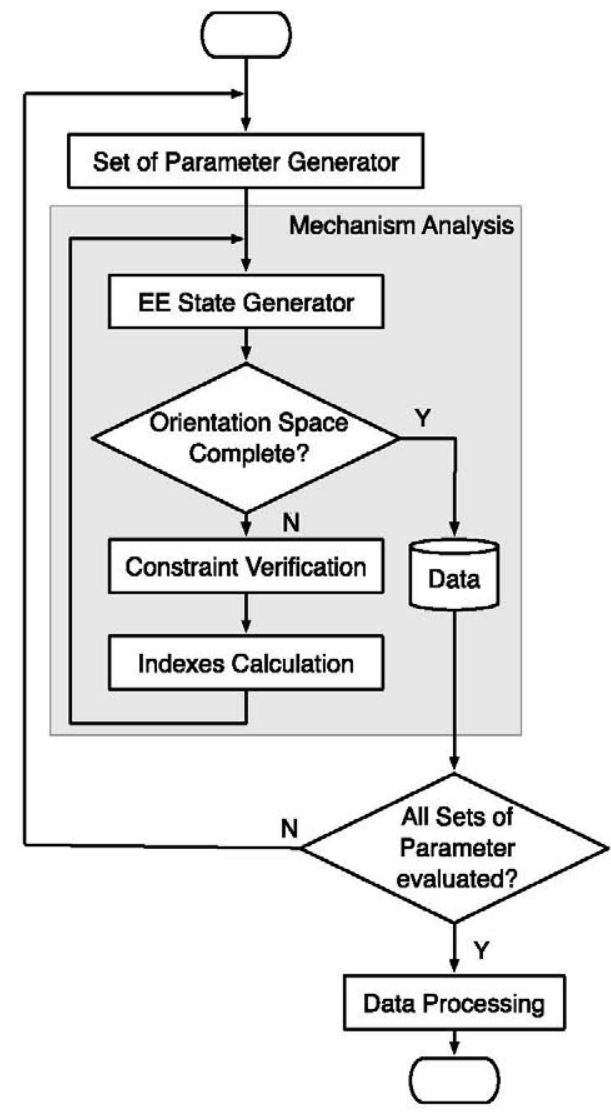

Fig. 4. Methodology implemented.

\subsection{End-effector state generator}

The parallel mechanism under analysis has a spherical motion pattern, therefore the end-effector state generator generates all the possible orientations in $\mathbb{R}^{3}$.

The orientation generator uses the quaternion representation method for orientation, which permits a singularity-free representation. Furthermore, with the parameterization stated below, it permits a unique representation for each orientation without ambiguities, and all the orientation in $\mathbb{R}^{3}$ can be visualized as a solid sphere [18].

Therefore, a given rotation represented by a unit axis $\hat{\boldsymbol{u}}$ and the quantity $\phi$ rotated around this axis, can also be expressed as a unit quaternion, as follows:

$\boldsymbol{p}=\left[\begin{array}{llll}e_{0}, & e_{1}, & e_{2}, & e_{3}\end{array}\right]^{T}=\left[\begin{array}{ll}\cos (\phi / 2), & \hat{\boldsymbol{v}} \sin (\phi / 2)\end{array}\right]^{T}$,

where (15) must satisfy: $\boldsymbol{p} \boldsymbol{p}^{T}=\|\boldsymbol{p}\|^{2}=e_{0}{ }^{2}+e_{1}{ }^{2}+e_{2}{ }^{2}+e_{3}{ }^{2}=1$ and $\|\hat{\boldsymbol{v}}\|=1$.

The vector $\boldsymbol{s}=\hat{\boldsymbol{v}} \sin (\phi / 2)$, will describe a solid sphere $\boldsymbol{S}$ of unitary radius. Therefore, a point of the solid sphere $\boldsymbol{s}=$ $\left[e_{1}, e_{2}, e_{3}\right]$, defines a vector where its direction is associated to the rotation axis according to $\hat{\boldsymbol{v}}=\boldsymbol{s} /\|\boldsymbol{s}\|$, and its norm is related to the angle of rotation according to $\phi= \pm 2 \arcsin (\|\boldsymbol{s}\|)$. The \pm sign depends on whether $e_{3} \geq 0$ or $e_{3}<0$, respectively. The $z$-coordinate of the direction vector $\hat{\boldsymbol{v}}=\left[v_{x}, v_{y}, v_{z}\right]$ is considered always $v_{z} \geqslant 0$ in order to avoid ambiguities in the orientation representation.

Therefore, the orientation generator defines a vector $s=$ $\left[e_{1}, e_{2}, e_{3}\right]$, where $e_{1}, e_{2}$ and $e_{3} \in\left[\begin{array}{ll}-1, & 1\end{array}\right]$. Thus, if $\|s\| \leqslant 1$ (i.e. $s$ belongs to the spherical solid of unitary radius), then the orientation generated will be given as follows:

$\boldsymbol{p}^{T}=\left[\begin{array}{ll}\sqrt{1-\|\boldsymbol{s}\|^{2}}, & \boldsymbol{s}\end{array}\right]^{T}=[\cos (\phi / 2), \quad \hat{\boldsymbol{v}} \sin (\phi / 2)]^{T}$.

\subsection{Constraints verification}

The workspace of a mechanism is always dependent to its physical limitations, which are given by the implicit constraints of the kinematic chains, such as the range of work of each joint and the collisions or interferences between the elements of the mechanism, among others. In this work, the physical limitations of the PM under evaluation are considered and modeled as follows:

1. Prismatic Stroke Constraint: the prismatic stroke constraint imposed on each leg can be written as $d_{\min } \leq d_{i} \leq d_{\max }$. Where $d_{i}$ is the state of the prismatic joint of the ith-limb given by (4), and $d_{\min }$ and $d_{\max }$ are the minimum and maximum strokes of the actuator. During the simulations, it is assumed that $d_{\min }=0$ and $d_{\max }=2 R_{B}$.

2. Universal and Spherical Joint Constraint: it is considered that its relative movement can never exceed the region limited by the circular cone defined by an axis normal to the joint, an aperture angle $\delta_{\max }=160^{\circ}$ and its apex located at the center of the joint ([19]).

3. Leg Interference: it is based on the evaluation of the minimum distance between two cylinders ([20]). In this work, it is only considered the interference between any link $L_{2}$ and link $L_{3}$, because the constraints imposed by the universal joints do not allow collisions between any other elements. The diameter of the cylinders for $L_{3}$ and $L_{2}$ are assumed as $d_{L_{3}}=0.1 R_{B}$ and $d_{L_{2}}=0.15 R_{B}$, respectively.

\subsection{Index calculation}

\subsubsection{Workspace index}

The workspace of the mechanism is defined by all the positions and orientations that can be achieved without exceeding its physical limitations.

The simpler way to obtain the workspace is to discretize the objective workspace into nodes $\left(n_{G}\right)$, and identify those nodes $\left(n_{\text {WS }}\right)$ that are feasible. Therefore, the ratio:

$W S I=\frac{n_{W S}}{n_{G}}$

gives an idea of the workspace capabilities of the mechanism, and can only take values in the interval $[0,1]$.

However, since there are singular configurations that separate the workspace into smaller regions (as it is presented in the results section), in this work the evaluation of the WSI only considers those nodes that belongs to the maximum region free of singular postures.

\subsubsection{Global condition index}

In linear algebra, the condition number expresses the sensitivity of the solution of a linear system to errors in the known data or the system itself. On the other hand, the Jacobian matrix of a robot maps the joint space into the end effector space. Therefore, the performance of a robot for a given configuration can be characterized with the condition number of the Jacobian matrix [5], according to $\kappa(J)=|J| \cdot J^{-1} \mid$, where

$|J|=\max _{\boldsymbol{x} \rightarrow 0} \frac{\|\boldsymbol{J} \boldsymbol{x}\|}{\|\boldsymbol{x}\|}$.

Since the condition number can take values in the interval $[1, \infty)$, the condition index given by $C I(J)=\kappa^{-1}(J)$ and bounded to $(0,1]$ is usually used instead. When it tends to zero, the Jacobian Matrix is badly conditioned, and the mechanism could fall into a singular configuration. 


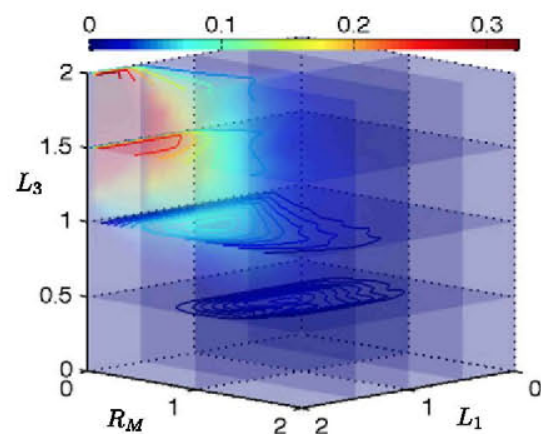

(a) WS performance.

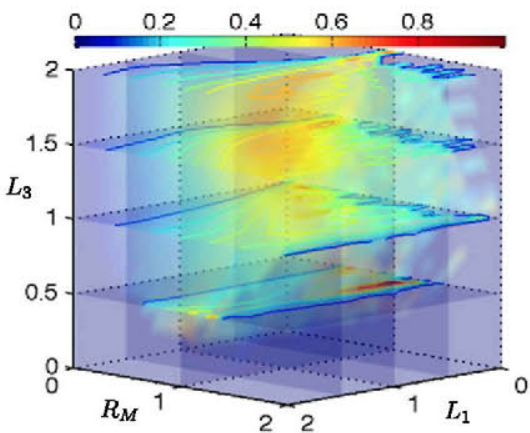

(b) GCI performance.

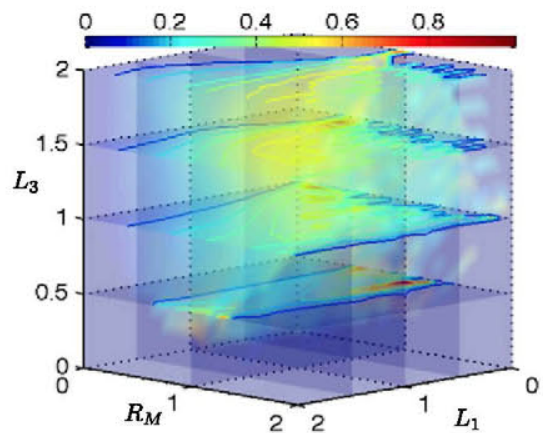

(c) $R_{C I}$ performance.

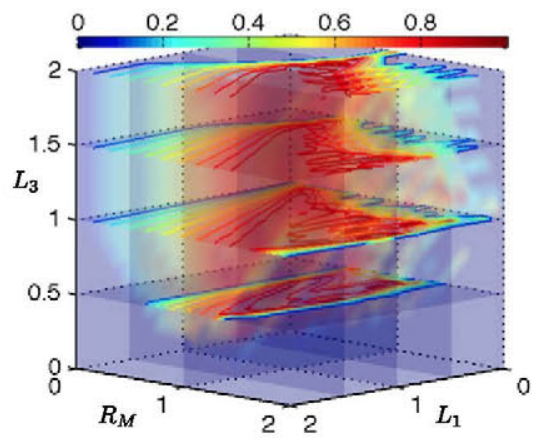

(d) $\mathrm{KCl}$ performance.

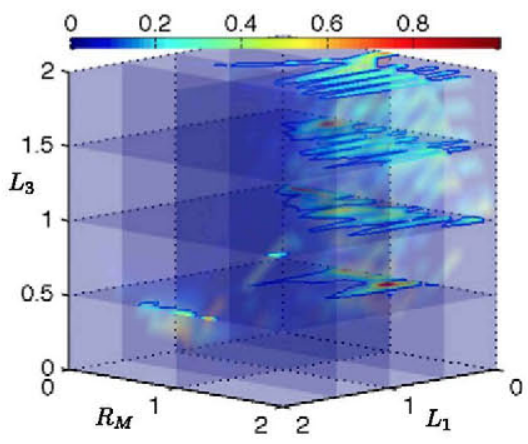

(e) GII performance.

Fig. 5. Performance of the PM according to its design parameters.

The global condition index [21], is introduced in order to evaluate the global performance of the mechanism in all its workspace and it is defined as follows:

$G C I=\frac{\int_{W} C I(J) d W}{\int_{W} d W}$

and takes values over the interval $(0,1]$. However, given the complexity in finding an exact solution for the integral term, a discrete formulation is used instead:

$G C I=\frac{\sum_{j=1}^{n_{W S}} C I(J)}{n_{W S}}$,

where $n_{W S}$ are the nodes that belong to the workspace of the PM.

\subsubsection{Global level and distribution ratio index}

The overall performance of the mechanism can be characterized by means of the $G C I$, though, this index does not reflect the uniformity or non-uniformity of the performance of the mechanism. If the discrete formulation for the $G C l$ is observed comprehensively, it resembles the mean value for the $\mathrm{Cl}$ over the workspace of the mechanism $\left(n_{W S}\right)$. Thus, the GCI tolerates small regions of poor behavior and may overrate a design that is unacceptable in practice.

Therefore, a novel bounded index named Level and Distribution Ratio Index $\left(R_{C}\right)$ is proposed in this work. It penalizes the nonuniformity of the index over the workspace, defined as follows,

$R_{C I}=\frac{G C I}{\alpha_{4} \sigma_{C I}+1}$,

where $\sigma_{C I}$ is the standard deviation of the $\mathrm{Cl}$ over the workspace, and $R_{C} \in(0,1] . \alpha_{4}$ is a ponderation factor.

\subsubsection{Kinematic conditioning index}

The Kinematic Conditioning Index $(\mathrm{KCI})$, is defined as follows [22]:
$K C I=\frac{1}{\kappa_{\min }(J)}$,

where $\kappa_{\min }(J)$ is the minimum condition number of the Jacobian matrix in the entire workspace. This index gives an idea of the worst performance of the mechanism in the workspace, and $\mathrm{KCI} \in$ $[0,1]$. A PM with $K C I=1$ means that it is isotropic since all their singular values are identical, while a $\mathrm{KCI}=0$ means that the PM possesses at least one singular posture in its workspace. Hence, it can be inferred that a higher $\mathrm{KCI}$ makes a matrix closer to the isotropic condition and a lower $\mathrm{KCl}$ makes it closer to singularity.

\subsubsection{Global isotropy index}

The Global Isotropy Index (GII) was introduced by Stocco et al. [23] and defined as the relationship between the largest and smallest singular values of the Jacobian matrix in the entire workspace, as follows:

$\operatorname{GII}(J)=\frac{\lambda_{\min }(J)}{\lambda_{\max }(J)}$.

The GII is a worst-case performance measure. The GII is essentially a global inclusive version of the condition number. However, the GII evaluates a robot design by the bounds on its singular values and not by an average value. It does not tolerate intermittent displays of poor performance. It also takes scale information into account since this information is reflected by the singular value bounds.

\section{Simulation results}

For the dimensional synthesis, the PMs are parametrized considering the radius of the base platform $\left(R_{B}\right)$ as the normalizing length, and the rest of its elements will be expressed as an $R_{B}$ times scale factor. In this work, 9261 PMs are generated and parametrized considering the scale factors presented in Table 1. 


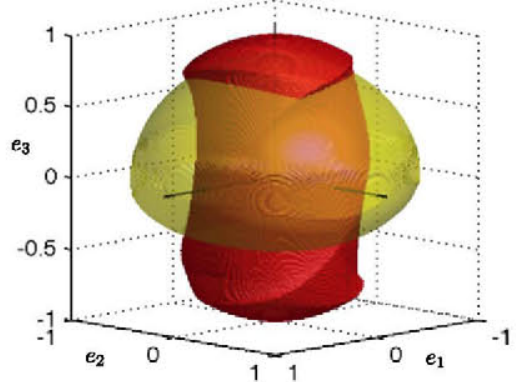

(a) $P M^{1}$ workspace and singular surface.

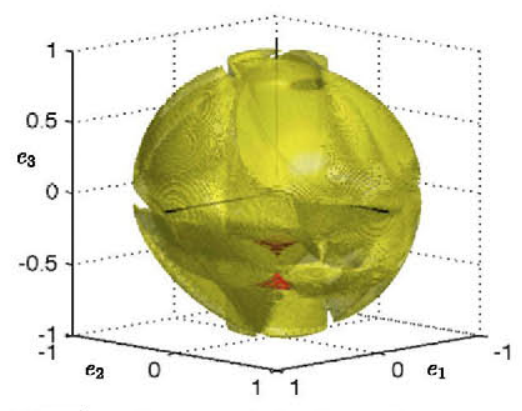

(d) $P M^{2}$ workspace and singular surface.

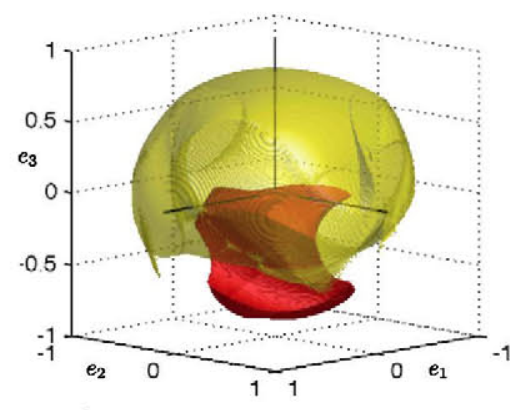

(g) $P M^{3}$ workspace and singular surface.

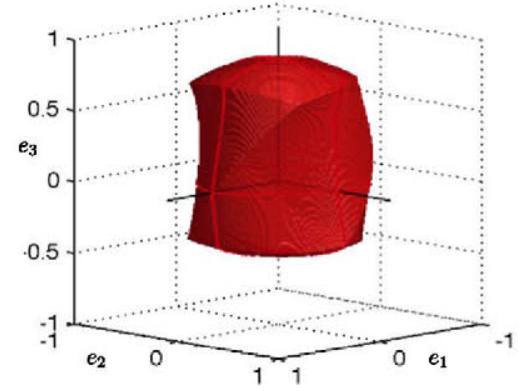

(b) $P M^{1}$ workspace free of singularities.

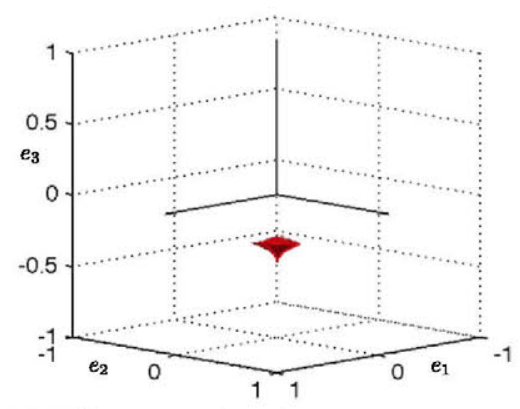

(e) $P M^{2}$ workspace free of singularities.

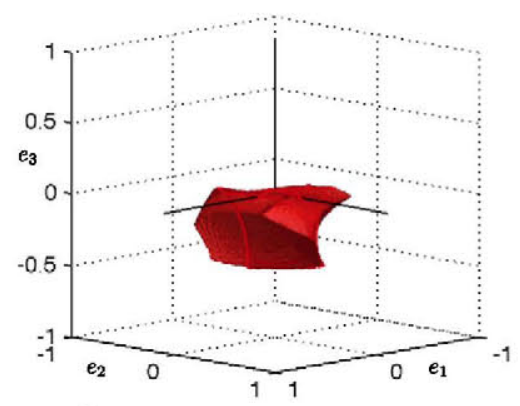

(h) $P M^{3}$ workspace free of singularities.

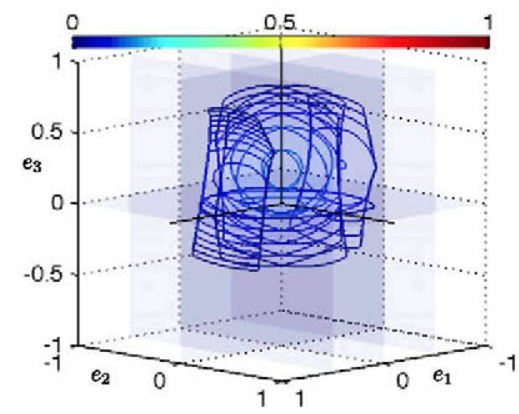

(c) $P M^{1}$ local dexterity.

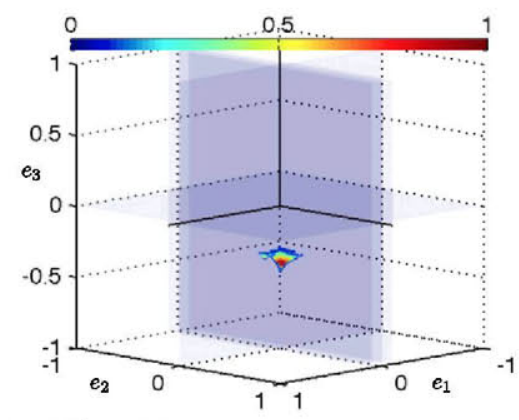

(f) $P M^{2}$ local dexterity.

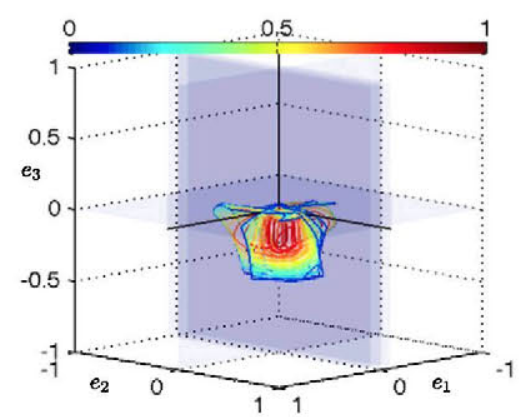

(i) $P M^{3}$ local dexterity.

Fig. 6. Local characteristics of the PMs with maximum indexes.

Table 1

Parametrization of the PM.

\begin{tabular}{lll}
\hline Parameter & Scale factor & Variation \\
\hline$R_{B}$ & 1 & - \\
$R_{M}$ & {$[0,2]$} & 0.1 \\
$L_{1}$ & {$[0,2]$} & 0.1 \\
$L_{2}$ & 0.25 & - \\
$L_{3}$ & {$[0,2]$} & 0.1 \\
$L_{4}$ & 0 & - \\
\hline
\end{tabular}

The objective workspace analyzed is discretized into 33401 states nodes. The performance of the PMs in terms of each global index is presented in Fig. 5 as contoured slices.

As shown in Fig. 5(a), the workspace of the mechanism increases as $L_{3}, L_{1}$ tends to 2 , and $R_{M}$ tends to 0 . It can also be observed that the WS of the PM is drastically reduced if $L_{3}$ decreases. Fig. 5(b) shows that a PM will have a good performance in terms of the GCI if all the parameters tend to 2 . However, it also shows that enhanced performances can be achieved if $L_{1}$ and $L_{3}$ tend to 0.5 and $R_{M}$ tends to 1. As shown in Fig. 5(c), the evaluation of the PMs in terms of $R_{C I}$ resembles the behavior of $G C I$, but with lower intensities. Therefore, all the PMs present a uniform distributed performance. In Fig. 5(d), it can be observed that the $\mathrm{KCI}$ is enhanced if $R_{M}, L_{1}$ and $L_{3}$ tend to 2 . However, good performances in terms of $K C I$ can be obtained if $R_{M}$ and $L_{1}$ tend to 0 and $L_{3}>0$. Fig. 5(e) presents the performances of the PMs in terms of GII. It can be seen that in order to achieve a good performance, $L_{1}$ must tend to 0 , and $R_{M}$ must be related to $L_{3}$ as follows: while $L_{3}$ tends to $1, R_{M}$ must tend to 0 .

The PMs with maximum performances found in terms of each global index are presented in Table 2. As it can be observed, there are three optimum PMs: $P M^{1}=[0.2,2.0,2.0], P M^{2}=$ $[1.2,0.2,0.5]$ and $P M^{3}=[1.4,1.7,1.3] . P M^{2}$ is optimum in terms of the $G C I, R_{C I}$, and $G I I$ indexes, however it has a very reduced workspace. On the other hand, the optimum PM in terms of WSI (i.e. $P M^{1}$ ) has a poor performance in terms of the other indexes. These PMs are further analyzed by evaluating their workspaces, their singular configurations and their local dexterity $(\mathrm{CI}(J))$.

The workspace of the PMs and their singular configurations are presented in Figs. 6(a), (d), (g). The workspace is presented as a solid volume, while the singular configurations (i.e. those configurations where $\operatorname{det}(J)=0$ ) as a translucent surface. The workspace of the PMs free of singularities are presented in Figs. 6(b), (e), (h), as a solid volume. The local dexterity of PMs are presented as colored contours for different slices of their workspace free of singularities in Figs. 6(c), (f), (i).

From the previous results, it can be easily observed that the optimum PMs in terms of each GPI do not have a wide non 


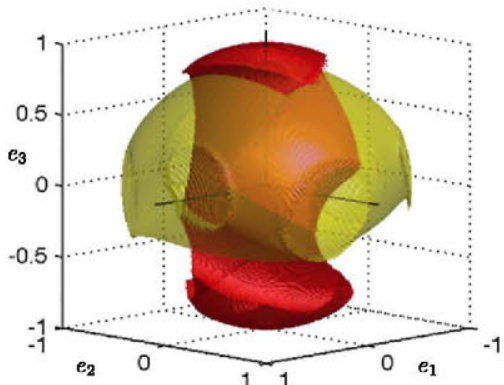

(a) $P M^{4}$ workspace and singular surface.

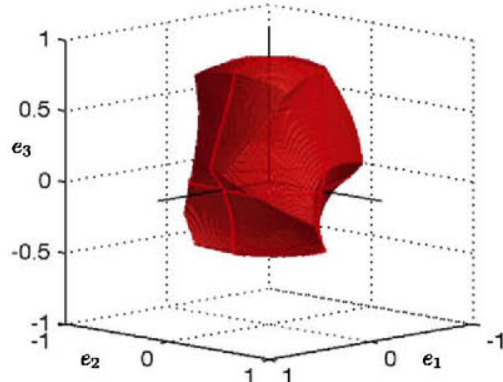

(b) $P M^{4}$ workspace free of singularities.

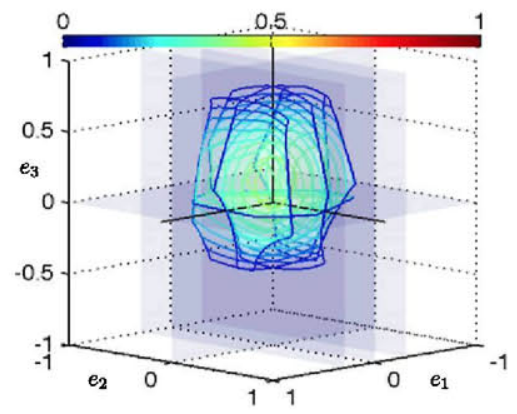

(c) $P M^{4}$ local dexterity.

Fig. 7. Local characteristics of the PMs with maximum indexes.

Table 2

PMs with maximum performance indexes.

\begin{tabular}{llllllll}
\hline Index & WSI & $G C I$ & $R_{C}$ & $K C I$ & $G I$ & $C$ & {$\left[R_{M}, L_{1}, L_{3}\right]$} \\
\hline WSI $_{\max }$ & 0.3231 & 0.0708 & 0.0686 & 0.1254 & $4.5 \mathrm{e}-4$ & 0.2287 & {$[0.2,2.0,2.0]^{1}$} \\
$G C I_{\max }$ & $2.39 \mathrm{e}-4$ & 0.9992 & 0.9992 & 0.9992 & 0.9992 & 0.0024 & {$[1.2,0.2,0.5]^{2}$} \\
$R_{C \max }$ & $2.39 \mathrm{e}-4$ & 0.9992 & 0.9992 & 0.9992 & 0.9992 & 0.0024 & {$[1.2,0.2,0.5]$} \\
$K C I_{\max }$ & 0.0480 & 0.5328 & 0.4463 & 0.9999 & 0.0051 & 0.2556 & {$[1.4,1.7,1.3]^{3}$} \\
$G I I_{\max }$ & $2.39 \mathrm{e}-4$ & 0.9992 & 0.9992 & 0.9992 & 0.9992 & 0.0024 & {$[1.2,0.2,0.5]$} \\
\hline$C$ & 0.2276 & 0.2499 & 0.2270 & 0.4385 & 0.0050 & 0.5688 & {$[0.7,2.0,1.8]^{4}$} \\
\hline
\end{tabular}

singular workspace and good local dexterity at the same time. Thus, the performance of the PMs has to be evaluated in terms of an index that integrates all the GPIs. Therefore, the compound index presented in Section 3, is defined as:

$C=10 W S I\left(R_{C I}+K C I+G I\right)$.

It must be noticed in (24) that the $G C I$ is not included in the definition of the compound index in order to avoid redundancy of information since the performance of the PMs in terms of $R_{C I}$ resembles the behavior of the $G C I$.

The best PM in terms of the compound index is given by $P M^{4}=$ $[0.7,2.0,1.8]$. In Fig. 7 , an insight of the characteristics of this PM is presented. As it can be observed, it has a workspace greater than $22 \%$ of all the 3D orientation space, and it also presents an acceptable local dexterity, (see Table 2).

\section{3PSU-1S real prototype}

Considering the performance of $P M^{4}$ obtained in the previous section, and a radius of the base platform $R_{B}=50 \mathrm{~mm}$, the first prototype of the 3PSU-1S PM is implemented. (See Fig. 8).

The 3PSU-1S spherical PM uses 3 Faulhaber Linear DCServomotors LM 1247 080-01 for the actuation. These linear motors are made up of a magnetized rod that slides inside a controlled magnetic field, providing a controlled linear motion.

Given the reduced range of work of the commercially available spherical joints, the spherical joints of the mechanism are implemented as a universal joint plus a revolute joint orthogonally placed. The kinematic model of the mechanism explained in Section 2 is implemented in Labview. The linear motors are commanded via a CAN bus.

The maximum pure rotations along the principal axis of the mechanism (i.e. $\boldsymbol{x}, \boldsymbol{y}, \boldsymbol{z}$ ) are presented in Fig. 9. The maximum pure rotations along $\boldsymbol{z}$, are limited by the singular configuration of the mechanism.

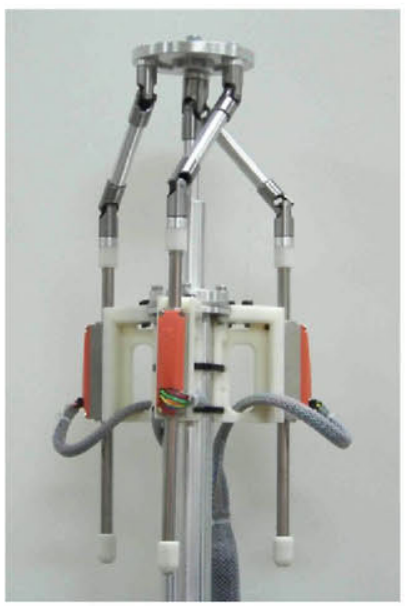

Fig. 8. 3PSU-1S real prototype.

\section{Conclusions and discussion}

In this work, the dimensional synthesis for the 3PSU-1S spherical PM has been presented.

The PM is parametrized and normalized according to a geometrical characteristic. This consideration allows a dimensionless analysis, which permits a scalable solution that can easily be implemented in a real prototype.

The criteria adopted for the dimensional synthesis is based on the evaluation of a compound performance index that integrates the workspace and dexterity characteristics of the PM. In this work, it has been proven that the dimensional synthesis of a mechanism cannot be based on the evaluation of only one design criteria, but it has to integrate different perspectives for the evaluation.

The methodology presented in this work could be used as a guide for the design of spherical parallel manipulator, which could be extended to a spatial parallel mechanism if some criteria for the homogenization of the Jacobian matrix is adopted. 


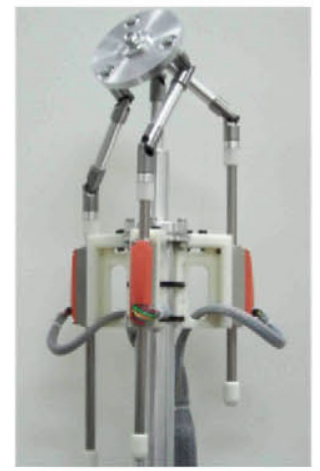

(a) $R x_{\min }=-30^{\circ}$.

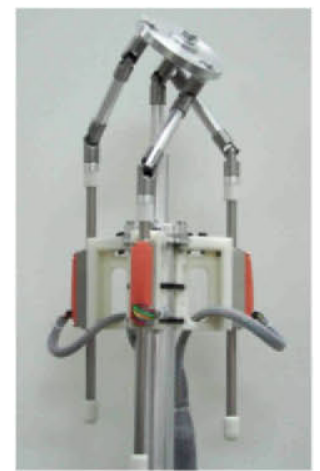

(d) $R y_{\max }=30^{\circ}$.

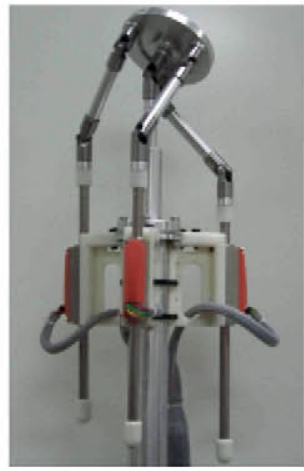

(b) $R x_{\max }=30^{\circ}$.

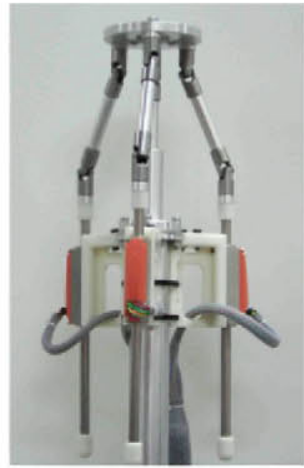

(e) $R z_{\min }=-30^{\circ}$.

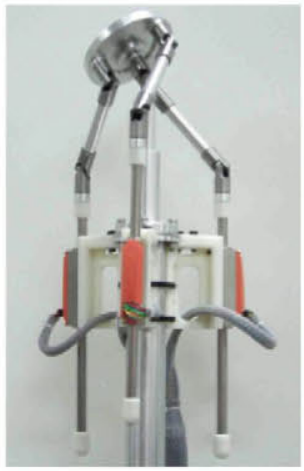

(c) $R y_{\min }=-30^{\circ}$.

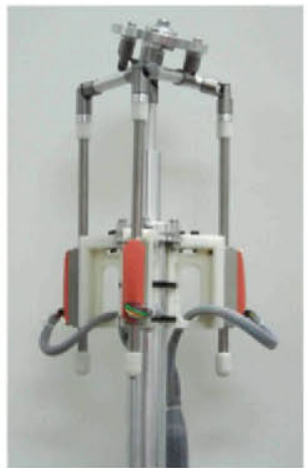

(f) $R z_{\max }=60^{\circ}$

Fig. 9. 3PSU-1S maximum pure rotation.

\section{Acknowledgments}

The first author belongs to the MAEC-AECID grant program. The fourth author is an Assistant Professor from the Universidad Nacional de Colombia and grant COLCIENCIAS.

\section{References}

[1] X. Kong, C. Gosselin, Type Synthesis of Parallel Mechanisms, in: Springer Tracts in Advanced Robotics, vol. 33, Springer, Berlin, Heidelberg, 2007.

[2] G. Gogu, Structural Synthesis of Parallel Robots: Part 1: Methodology, Springer Verlag, 2007.

[3] J.M. Herve, The Lie group of rigid body displacements, a fundamental tool for mechanism design, Mechanism and Machine Theory 34 (5) (1999) $719-730$.

[4] J.P. Merlet, in: G.M.L. Gladwell (Ed.), Parallel Robots, second ed., in: Solid Mechanics and its Applications, vol. 128, Springer-Verlag, Berlin/Heidelberg, 2006, p. 417. http://dx.doi.org/10.1007/1-4020-4133-0.

[5] O. Ma, Optimum architecture design of platform manipulators, Advanced Robotics, 1991. 'Robots in Unstructured Environments', 91 ICAR., Fifth International Conference on, vol. 2, 19-22 June 1999, pp. 1130-1135.

[6] C. Gosselin, J. Angeles, The optimum kinematic design of a planar threedegree-of-freedom parallel manipulators, Journal of Mechanisms, Transmissions and Automation in Design, Trans. ASME 110 (1) (1988) 35-41.

[7] C. Gosselin, J. Angeles, The optimum kinematic design of a spherical three Degree-of-Freedom parallel manipulator, Journal of Mechanisms, Transmissions and Automation in Design, Trans. ASME 111 (2) (1989) 202-207.

[8] X. Jun Liu, Optimum design of 3-DOF spherical parallel manipulators with respect to the conditioning and stiffness indices, Mechanism and Machine Theory 35 (9) (2000) 1257-1267.

[9] L.A. Silva, J.M. Sebastian, R. Saltaren, R. Aracil, J. San Pedro, RoboTenis: optimal design of a parallel robot with high performance, 2005 IEEE/RSJ International Conference on Intelligent Robots and Systems, 2005, pp. 2134-2139.

[10] T. Huang, M. Li, X.M. Zhao, J.P. Mei, D.G. Chetwynd, S.J. Hu, Conceptual design and dimensional synthesis for a 3-DOF module of the TriVariant-a novel
5-DOF reconfigurable hybrid robot, IEEE Transactions on Robotics 21 (3) (2005) 449-456

[11] S. Lee, S. Kim, Analysis and Optimal Design of a New 6 DOF Parallel Type Haptic Device, 2006 IEEE/RSJ International Conference on Intelligent Robots and Systems, Octber 2006, pp. 460-465.

[12] M. Badescu, C. Mavroidis, Workspace optimization of 3-legged UPU and UPS parallel platforms with joint constraints, Journal of Mechanical Design $126(2)$ (2004) p. 291.

[13] Y. Lou, G. Liu, Z. Li, Randomized optimal design of parallel manipulators IEEE Transactions on Automation Science and Engineering 5 (2) (2008) 223-233.

[14] M. Zoppi, D. Zlatanov, R. Molfino, On the velocity analysis of interconnected chains mechanisms, Mechanism and Machine Theory. 41 (11) (2006) 1346-1358.

[15] I.A. Bonev, D. Zlatanov, C.M. Gosselin, Singularity analysis of 3-DOF planar parallel mechanisms via screw theory, Journal of Mechanical Design, ASME 125 (3) (2003) 573-581. http://dx.doi.org/10.1115/1.1582878.

[16] D. Zlatanov, I.A. Bonev, C.M. Gosselin, Constraint Singularities of Parallel Mechanism, 2002 IEEE International Conference on Robotics \& Automation, http://dx.doi.org/10.1109/ROBOT.2002.1013408.

[17] LW. Tsai, Robot Analysis:The Mechanics of Serial and Parallel Manipulators, John Wiley \& Sons, Inc, 1999.

[18] J.M. Sabater, N. Garcia, C. Perez, J.M. Azorin, R. Saltaren, E. Yime, Design and analysis of a spherical humanoid neck using screw theory, The First IEEE/RASEMBS International Conference on Biomedical Robotics and Biomechatronics, 2006. BioRob 2006, 1166-1171.

[19] K. Oen, L. Wang, Extreme reaches and maximal reachable workspace for rotary tools mounted on a Stewart platform manipulator, Journal of the Chinese Institute of Engineers 29 (6) (2006) 967-974.

[20] J.P. Merlet, D. Daney, Legs Interference Checking of Parallel Robots over a Given Workspace or Trajectory, Robotics and Automation, Orlando, Florida, 2006, no. May, pp. 757-762.

[21] C. Gosselin, J. Angeles, A global performance index for the kinematic optimization of robotic manipulators, Journal of Mechanical Design 113 (3) (1991) 220.

[22] J. Angeles, Fundamentals of Robotic Mechanical Systems: Theory, Methods and Algorithms, Springer New York, New York, NY, 2003.

[23] L]. Stocco, S.E. Salcudean, F. Sassani, Optimal kinematic design of a haptic pen, IEEE/ASME Transactions on Mechatronics 6 (3) (2001) 210-220. 


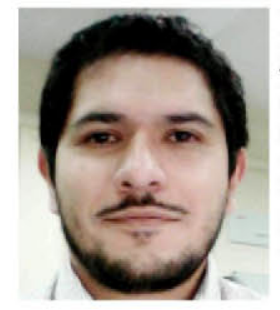

Lisandro J. Puglisi received his B.S. degree in electronic engineering in 2006 from the Universidad Nacional de Tucumán, Argentina, and his M.S. degree in Automation and Robotics from the Universidad Politécnica de Madrid, Spain, in 2010. He is currently working toward a Ph.D. at the Centre for Automation and Robotics of the UPMCSIC, in Spain. His research interests include kinematics, dynamics, control of robotic parallel mechanisms and hydraulic robots applications.

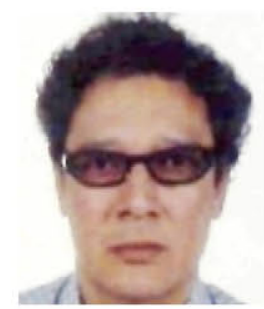

Roque J. Saltaren received his degree in Mechanical Engineering from the Universidad del Valle, Cali, Colombia in 1980, his M.Sc. degree in Electrical Engineering from the Universidad de los Andes, Bogotá, Colombia in 1990, and his Ph.D. in Industrial Engineering from the Universidad Politécnica de Madrid, Madrid, Spain in 1996. He is currently Titular Professor at the Universidad Politécnica de Madrid, Spain. He is also a Research Assistant of the Centre for Automation and Robotics of Universidad (UPM-CSIC). His research interests include parallel robots, robotics, and life science and robotics industrial applications. He has chaired several international conferences and workshops in robotics and parallel robots.

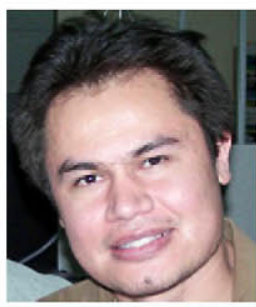

Hector A. Moreno received his B.S, degree in Mechanical Engineering in 2004 and M.S. degree in Electrical Engineering in 2006, both from the Instituto Tecnologico de la Laguna, Mexico. He earned a M.S, degree in Automation and Robotics from the Universidad Politécnica de Madrid (UPM), Spain, in 2009. He is currently working toward a Ph.D. at the Centre for Automation and Robotics of the UPM-CSIC, in Spain. His research interests include the kinematics, dynamics and control of robotic mechanical systems.

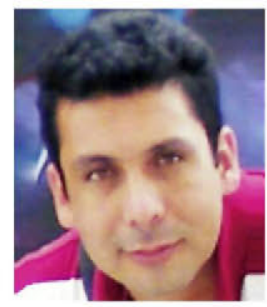

Pedro F. Cárdenas received his B.S. degree in Electronics from Universidad Pedagógica y Tecnológica de Colombia (UPTC) in 2000, and his M.S. degree in Industrial automation from the Universidad Nacional de Colombia (UNC) Bogotá in 2008. He is an Assistant Professor at the Universidad Nacional de Colombia, Bogotá. He is currently working toward a Ph.D in the Centre for Automation and Robotics of Universidad Politecnica de Madrid-CSIC. Spain. His research interests include kinematics, dynamic control of robotic parallel mechanisms, and surgery robotics.

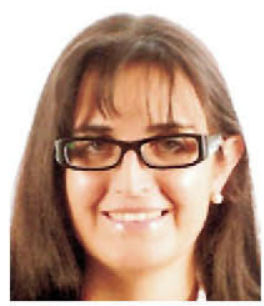

Cecilia Garcia received her Ph.D. from Universidad Nacional de San Juan, Argentina in 2000. She is currently an Assistant Professor at the Universidad Politécnica de Madrid, Spain. She is also a Research Assistant of the Centre for Automation and Robotics of the Universidad. Her research interests include telerobotics, artificial intelligence, robotics, and life science. She has chaired several international conferences and workshops in robotics, telerobotics, parallel mechanisms, and multiagent systems.

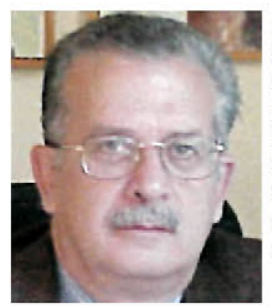

Rafael Aracil received his degree in Electrical Engineering in 1971 and his Ph.D. in Engineering in 1975, both from the Escuela Téccnica Superior de Ingenieros Industriales, Madrid, Spain. Currently, he is a Full Professor and Head of the Group of Robots and Intelligent Machines, at Universidad Politécnica de Madrid, Escuela Técnica Superior de Ingenieros Industriales, Madrid, Spain. His main activities have been in the area of advanced manufacturing, robotics, and image processing. 